\title{
A Linear Relationship Between a Body Shape Index and Risk of Incident Type 2 Diabetes: A Secondary Analysis Based on a Retrospective Cohort Study in Japan
}

This article was published in the following Dove Press journal:

Diabetes, Metabolic Syndrome and Obesity: Targets and Therapy

\author{
Wei Zhaol,* \\ Jing-Jing Tong ${ }^{2, *}$ \\ Yong-Tong Cao' \\ Jing-Hua $\mathrm{Li}^{\prime}$
}

'Department of Clinical Laboratory, China-Japan Friendship Hospital, Beijing I00029, People's Republic of China; ${ }^{2}$ Liver Failure Treatment and Research Center, The Fifth Medical Center of PLA General Hospital, Beijing 100039 ,

People's Republic of China

*These authors contributed equally to this work

Correspondence : Jing-Hua Li; Yong-Tong

Cao

Department of Clinical Laboratory,

China-Japan Friendship Hospital, No. 2

Yinghua Road, Chaoyang District, Beijing 100029, People's Republic of China

Tel +86 I08420 5486; +86 I084205580

Email lijinghua620524@I63.com;

caoyongtong100@sina.com
Purpose: This study aimed to evaluate the association between a body shape index (ABSI) and incident type 2 diabetes and to explore the shape of their relationship in a cohort of Japanese adults.

Patients and Methods: Data from 15,462 Japanese adults aged 18-79 years attending the NAGALA study (NAfld in the Gifu Area, Longitudinal Analysis) were used. Body weight, height, and waist circumference were measured. Blood samples were measured for serum lipid, glucose, and HbAlc. The risk of incident type 2 diabetes according to ABSI was estimated using multivariate Cox regression models. We examined a potential nonlinear relationship using a smoothing function analysis. Subgroup analyses were conducted according to age, gender, smoking status, alcohol intake, fatty liver, and BMI.

Results: After adjusting for potential confounding factors (age, gender, smoking status, alcohol intake, fatty liver, systolic blood pressure, BMI, fasting plasma glucose, HbA1c, HDL-cholesterol, triglycerides), a linear relationship was observed between ABSI and risk of type 2 diabetes. The hazard ratio (HR) and 95\% confidence intervals $(95 \% \mathrm{CI})$ for incident type 2 diabetes with ABSI $\left(10^{-2} \mathrm{~m}^{11 / 6} \mathrm{~kg}^{-2 / 3}\right)$ were $1.51(1.13,2.01)(p=0.005)$. When ABSI was handled as categorical variable, the HRs and $95 \%$ CIs in the quartile 2 to 4 versus the quartile 1 were $0.97(0.67,1.41), 1.21(0.85,1.72)$ and $1.30(0.92,1.83)$, respectively (P for trend $=0.046)$. Subgroup analyses showed that the association stably existed in different subgroups including gender, age, smoking status, alcohol intake, fatty liver, and BMI.

Conclusion: ABSI was linearly associated with an elevated risk of incident type 2 diabetes across the full range of ABSI, independent of gender, age, smoking status, alcohol intake, fatty liver, SBP, BMI, FPG, HbA1c, HDL-cholesterol, and triglycerides.

Keywords: ABSI, type 2 diabetes, linear relationship, a retrospective cohort study

\section{Introduction}

Diabetes mellitus is one of the fastest growing health challenges of the 21 st century. International Diabetes Federation reported that prevalence of diabetes mellitus worldwide was 9.3\% (a staggering 463 million people) among adults aged 20-79 years in 2019, and estimated that this figure will reach 700 million by $2045 .^{1}$ The unprecedented increase in the number of patients with type 2 diabetes is largely due to an upsurge of related risk factors, which include obesity, energy-dense diets and physical inactivity. ${ }^{2}$ 
Body mass index (BMI) has been widely used as an indicator of general obesity. Researches have showed that BMI is positively associated with risk for type 2 diabetes. ${ }^{3,4}$ It was also found that BMI, even within normal range, strongly predicts diabetes mellitus mortality. ${ }^{5}$ But BMI cannot reflect body fat distribution. ${ }^{6}$ Clinical evidence suggests that central obesity is more strongly associated with diabetes than general obesity. ${ }^{3} \mathrm{~A}$ body shape index (ABSI), which was developed by Krakauer NY in 2012, can indicate abdominal deposition of adipose tissue. ${ }^{7}$ For a given height and weight, high ABSI indicates higher waist circumference than expected. ${ }^{8}$ ABSI can predict mortality independently from BMI in Americans and Europeans ${ }^{9,10}$ with a positive linear relationship. ${ }^{10}$ Some studies suggest that there is a positive association between ABSI and high risk of incident type 2 diabetes. ${ }^{11-16}$ However, most of them are cross-sectional study, which cannot reveal the causal relationship. Besides, the nature of the dose-response relationship is uncertain, especially whether it is a linear or threshold effect.

Therefore, the objectives of this cohort study are to examine whether ABSI is an independent risk factors for incident type 2 diabetes and to characterize the nature of the dose-response relationship in detail.

\section{Materials and Methods}

\section{Data Source}

We obtained data from the "DRYAD" database (https:// datadryad.org/). This database permitted users to freely download the raw data. According to Dryad Terms of Service, we cited Dryad data set ${ }^{17}$ in the present study.

\section{Study Design and Participants}

This study was a secondary analysis of the open data from the NAGALA study (NAfld in the Gifu Area, Longitudinal Analysis). It was a longitudinal study at Medical Health Checkup Center of Murakami Memorial Hospital (Gifu, Japan) from 2004 to 2015. The details of the NAGALA study were described in the original article. ${ }^{18}$ Briefly, 20,944 participants who participated in medical examination program between 2004 and 2015 and finished at least a second exam were recruited. Finally, 15,462 participants were screened for data analysis according to exclusion criteria. Exclusion criteria: 1) had a heavy drinking habit $(\mathrm{n}=739), 2)$ had hepatitis $\mathrm{B}$ or $\mathrm{C}$ virus $(\mathrm{n}=416), 3)$ used medication at baseline examination $(\mathrm{n}=2321), 4)$ had type 2 diabetes $(\mathrm{n}=323)$ or impaired fasting glucose $(\mathrm{n}=808), 5)$ missed data of covariates, including alcohol intake, exercise, height or abdominal ultrasonography $(\mathrm{n}=863)$, 6) had incorrect data of waist circumference $(n=2)$. Okamura et al completed the entire study. According to their description, the study was approved by the ethics committee of Murakami Memorial Hospital and informed consent was obtained from each participant.

\section{Data Collection and Measurements}

A standardized self-administered questionnaire was designed to obtain the medical history and lifestyle characteristics of all participants, including smoking status, alcohol habits and physical activity.

Anthropometric measurements including weight, height, waist circumference (WC) and blood pressure were measured by competent nurses. Body mass index (BMI) was calculated as weight/square of height $\left(\mathrm{kg} / \mathrm{m}^{2}\right)$. ABSI was calculated using the following equation: $\mathrm{ABSI}=\mathrm{WC} /\left(\mathrm{BMI}^{2 / 3}\right.$ height $\left.^{1 / 2}\right)$, the units of ABSI are $\mathrm{m}^{11 / 6}$ $\mathrm{kg}^{-2 / 3}$. Abdominal ultrasonography was performed by trained technicians. According to the images, fatty liver was diagnosed by gastroenterologists without reference to other individual data of the participants.

Blood samples were collected from the participants after $8 \mathrm{~h}$ of fasting. Samples were centrifuged immediately and were stored at $-80^{\circ} \mathrm{C}$ until analysis. Fasting blood samples were analyzed for high-density lipoprotein cholesterol (HDL-C), total cholesterol, triglycerides, fasting plasma glucose (FPG) and hemoglobin Alc (HbAlc), et al.

\section{Definition of Type 2 Diabetes}

Type 2 diabetes was diagnosed based on one or more of the following previously validated criteria: self-reported diabetes, fasting plasma glucose $\geq 7.0 \mathrm{mmol} / \mathrm{L}, \mathrm{HbA} 1 \mathrm{c}$ $\geq 6.5 \%$, or the initiation of diabetes treatment. ${ }^{19}$

\section{Statistical Analysis}

Data are presented as mean \pm standard deviation (normal distribution) or median (quartile) (skewed distribution) for continuous variables, and as number (percentage) for categorical variables. The baseline characteristics of different ABSI groups were analyzed using One-Way Anova (normal distribution), Kruscal Whallis $\mathrm{H}$ (skewed distribution) and chi-square test (categorical variables). Multivariate cox regression models were performed to calculate hazard ratios (HRs) and 95\% confidence interval (95\% CI) for incident type 2 diabetes. Both non-adjusted and multivariableadjusted models were listed. Covariates that changed initial regression coefficients by at least $10 \%$ were considered as 
confounding factors. In this study, the Cox models were adjusted for age, gender, smoking status, alcohol intake, BMI, fatty liver, systolic blood pressure (SBP), HDL-C, triglycerides, FPG, HbAlc. Tests for trend were conducted with cox regression by entering the median value of each ABSI quartile as a continuous variable in the models. The smoothing function analysis using generalized additive model was fitted to model the potential nonlinear relationship between ABSI and incident type 2 diabetes. The subgroup analyses were performed using stratified Cox regression models. Interaction among subgroups was inspected by the likelihood ratio test.

All statistical analyses were performed with the statistical software packages $\mathrm{R}$ version 3.4.3 (The $\mathrm{R}$ Foundation, Vienna, Austria) and EmpowerStats (X\&Y Solutions, Inc., Boston, MA, USA). $P$-value $<0.05$ (twosided) was considered statistically significant.

\section{Results}

\section{Baseline Characteristics of Study Participants}

During a median 5.4 years follow-up, 373 subjects among the 15,462 participants developed type 2 diabetes. Baseline characteristics of all participants are shown in Table 1. The mean age was $43.71 \pm 8.90$ years and about $45.49 \%$ of the participants are female. The mean ABSI was $(7.56 \pm 0.40)$ $* 10^{-2} \mathrm{~m}^{11 / 6} \mathrm{~kg}^{-2 / 3}$. Participants with higher ABSI were more likely to be older, physically inactive and to be smokers (past and current); more likely to have fatty liver, higher BMI, greater waist circumference.

\section{Association Between ABSI and Incident Type 2 Diabetes}

We used Cox regression models to evaluate the association between ABSI and risk of incident type 2 diabetes. Table 2 shows the non-adjusted and adjusted models simultaneously. In the non-adjusted model, ABSI was positively associated with risk of type 2 diabetes [HR and 95\% CI=3.13 (2.44, 4.01), $p<0.0011$. After adjustment for age, gender, smoking status, alcohol intake, BMI, fatty liver, SBP, FPG, HbAlc, HDL-cholesterol, triglycerides, the HR and $95 \%$ CI for the risk of type 2 diabetes with ABSI was $1.51(1.13,2.01)$ $(p=0.005)$. For the purpose of sensitivity analysis, we also handled ABSI as categorical variable and found that the HRs and $95 \%$ CIs of ABSI quartile 2-4 were $0.97(0.67,1.41)$, $1.21(0.85,1.72)$ and $1.30(0.92,1.83)$ in the fully-adjusted model, respectively $(\mathrm{P}$ for trend $=0.046$ ).

\section{Threshold Effect Analysis of ABSI on Incident Type 2 Diabetes}

To evaluate the shape of the relationship between ABSI and incident type 2 diabetes, we used a smoothing function analysis (Figure 1). This analysis reveals a continuous positive association between ABSI and type 2 diabetes with increasing ABSI without a threshold effect after adjusting for age, gender, smoking status, alcohol intake, BMI, fatty liver, SBP, FPG, HbA1c, HDL-cholesterol, and triglycerides.

\section{Subgroup Analyses}

The subgroup analyses for the correlation between ABSI and incident type 2 diabetes were presented in Figure 2. The participants were divided into several subgroups according to gender, age, smoking status, alcohol intake, fatty liver, and BMI. The results showed that association between ABSI and incident type 2 diabetes stably existed in different subgroups. It was observed that the HRs for incident type 2 diabetes were higher in the participants who are female, non-smokers, have fatty liver or whose BMI $\geq 23 \mathrm{~kg} / \mathrm{m}^{2}$, although the test for interactions were not statistically significant $(\mathrm{P}$ for interaction $=0.3465,0.3560$, 0.1368 and 0.1092 , respectively).

\section{Discussion}

The population-based longitudinal study was to examine the relationship between ABSI and incident type 2 diabetes. As is shown, ABSI was associated with an elevated risk of the incidence of type 2 diabetes, independent of age, gender, smoking status, alcohol intake, BMI, fatty liver, SBP, FPG, HbA1c, HDL-cholesterol, and triglycerides. We further revealed that the relationship between ABSI and risk of type 2 diabetes is linear across the full range of ABSI. Sensitivity analysis showed that the relationship stably existed in different subgroups including gender, age, smoking status, alcohol intake, fatty liver, and BMI.

A body shape index (ABSI), which was developed by Krakauer and Krakauer in 2012, consists of waist circumference, height and weight. However, ABSI had little correlation with height, weight, or BMI. ${ }^{7}$ There are several researches that have reported the associations between ABSI and incident diabetes mellitus. ${ }^{12,15,16,20}$ Our findings with ABSI are consistent with those studies. To our knowledge, only four cohort studies have estimated the association of ABSI and diabetes mellitus. In a 15-year Chinese 
Table I Baseline Characteristics of Participants by Categories of ABSI in the NAGALA Study, 2004-20I5

\begin{tabular}{|c|c|c|c|c|c|}
\hline \multirow[t]{2}{*}{ Variable } & \multicolumn{4}{|l|}{ ABSI Quartiles } & \multirow[t]{2}{*}{$p$-value } \\
\hline & Quartile I & Quartile 2 & Quartile 3 & Quartile 4 & \\
\hline Participants (n) & 3866 & 3865 & 3865 & 3866 & \\
\hline Age (years) & $41.39 \pm 8.27$ & $42.21 \pm 8.32$ & $43.90 \pm 8.57$ & $47.33 \pm 9.22$ & $<0.001$ \\
\hline Female & 2433 (62.93\%) & 1536 (39.74\%) & 1399 (36.20\%) & 1666 (43.09\%) & $<0.001$ \\
\hline Smoking status & & & & & $<0.001$ \\
\hline Never & $2673(69.14 \%)$ & $2200(56.92 \%)$ & 2075 (53.69\%) & $2083(53.88 \%)$ & \\
\hline Past & $517(13.37 \%)$ & 704 (18.21\%) & 877 (22.69\%) & $853(22.06 \%)$ & \\
\hline Current & $676(17.49 \%)$ & $96 \mathrm{I}(24.86 \%)$ & $913(23.62 \%)$ & $930(24.06 \%)$ & \\
\hline Alcohol intake (g/week) & I (0-36) & I $(0-60)$ & $4.2(0-84)$ & $2.8(0-90)$ & $<0.001$ \\
\hline Fatty liver & & & & & $<0.001$ \\
\hline No & 3525 (91.18\%) & 3191 (82.56\%) & $3018(78.09 \%)$ & 2987 (77.26\%) & \\
\hline Yes & $34 \mathrm{I}(8.82 \%)$ & 674 (17.44\%) & 847 (21.91\%) & 879 (22.74\%) & \\
\hline Habit of exercise & & & & & $<0.001$ \\
\hline$<$ l/week & 3063 (79.23\%) & 3139 (8I.22\%) & $3233(83.65 \%)$ & 3319 (85.85\%) & \\
\hline$\geq$ I/week & $803(20.77 \%)$ & $726(18.78 \%)$ & $632(16.35 \%)$ & $547(14.15 \%)$ & \\
\hline BMI $\left(\mathrm{kg} / \mathrm{m}^{2}\right)$ & & & & & $<0.001$ \\
\hline Non-overweight $(\mathrm{BMI}<23)$ & $2830(73.20 \%)$ & 2454 (63.49\%) & $2352(60.85 \%)$ & $2426(62.75 \%)$ & \\
\hline Overweight (BMI $\geq 23,<25)$ & $553(14.30 \%)$ & $729(18.86 \%)$ & $803(20.78 \%)$ & 791 (20.46\%) & \\
\hline Obese $(\mathrm{BMI} \geq 25)$ & $483(12.49 \%)$ & $682(17.65 \%)$ & $710(18.37 \%)$ & 649 (16.79\%) & \\
\hline Waist circumference $(\mathrm{cm})$ & $69.79 \pm 7.55$ & $75.47 \pm 7.78$ & $78.68 \pm 8.03$ & $81.96 \pm 8.28$ & $<0.001$ \\
\hline ABSI $\left(10^{-2} \mathrm{~m}^{11 / 6} \mathrm{~kg}^{-2 / 3}\right)$ & $7.06 \pm 0.21$ & $7.44 \pm 0.07$ & $7.69 \pm 0.07$ & $8.07 \pm 0.21$ & $<0.001$ \\
\hline $\mathrm{SBP}(\mathrm{mmHg})$ & $110.98 \pm 14.26$ & $114.56 \pm 14.6 \mathrm{I}$ & $115.99 \pm 15.28$ & $116.46 \pm 15.10$ & $<0.001$ \\
\hline $\mathrm{DBP}(\mathrm{mmHg})$ & $69.04 \pm 9.88$ & $71.49 \pm 10.39$ & $72.82 \pm 10.72$ & $72.98 \pm 10.53$ & $<0.001$ \\
\hline Total cholesterol $(\mathrm{mmol} / \mathrm{L})$ & $5.02 \pm 0.83$ & $5.08 \pm 0.87$ & $5.16 \pm 0.88$ & $5.25 \pm 0.86$ & $<0.001$ \\
\hline HDL-cholesterol (mmol/L) & $1.56 \pm 0.40$ & $1.45 \pm 0.40$ & $1.41 \pm 0.39$ & $1.42 \pm 0.41$ & $<0.001$ \\
\hline Triglycerides (mmol/L) & $0.62(0.43-0.89)$ & $0.72(0.49-1.10)$ & $0.79(0.53-1.22)$ & $0.86(0.56-1.28)$ & $<0.001$ \\
\hline FPG (mmol/L) & $5.06 \pm 0.41$ & $5.17 \pm 0.40$ & $5.2 I \pm 0.4 I$ & $5.21 \pm 0.41$ & $<0.001$ \\
\hline $\mathrm{HbAlc}(\%)$ & $5.12 \pm 0.32$ & $5.17 \pm 0.32$ & $5.19 \pm 0.32$ & $5.21 \pm 0.33$ & $<0.001$ \\
\hline
\end{tabular}

Notes: Data presented are mean \pm SD, median (QI-Q3) or $\mathrm{N}(\%)$.

Abbreviations: BMI, body mass index; ABSI, a body shape index; SBP, systolic blood pressure; DBP, diastolic blood pressure; FPG, Fasting plasma glucose; HbAIc, hemoglobin Alc.

cohort study ( $\mathrm{n}=687)$, increasing the population ABSI by 1 SD would result in an increased $\mathrm{HR}(95 \% \mathrm{CI})$ of $41 \%$ $(12 \%, 77 \%) .{ }^{20}$ A cohort study with 37,581 participants in Japan followed for 4 years showed that z-score for ABSI was a predictor of diabetes with OR $(95 \% \mathrm{CI})=1.06(1.01$, 1.11). ${ }^{21}$ The Rural Chinese Cohort Study showed similar results. $^{16}$ However, a longitudinal ARIC study among white and black adults showed a different result. ${ }^{22}$ ABSI is not significantly associated with the risk for development of T2DM in White and Black males with the HR $(95 \% \mathrm{CI})=1.00(0.94,1.06)$ and $1.09(0.99,1.19)$ respectively. In our study, ABSI was positively associated with incident type 2 diabetes [HR and 95\% CI=1.51 (1.13, 2.01), $p=0.005]$. When ABSI was handled as categorical variable, the same trend was observed as well ( $\mathrm{p}$ for trend was 0.046). In a cohort of 46,651 European men and women, ABSI showed linear relationships with cardiovascular disease or all-cause mortality while BMI had a J-shaped relationship. ${ }^{10}$ However, there is no study to identify the shape of the association between ABSI and incident type 2 diabetes. In this study, we found a continuous positive linear association between ABSI and type 2 diabetes across the full range of ABSI.

Exploration of subgroup analysis is crucial for scientific researches. $^{23}$ In this study, we used gender, age, smoking status, alcohol intake, fatty liver and BMI as stratification variables and the subgroup analyses revealed that the association between ABSI and risk of type 2 diabetes stably existed in 
Table 2 Association Between ABSI and Incident Type 2 Diabetes in the NAGALA Study, 2004-20I5

\begin{tabular}{|c|c|c|c|c|c|c|c|c|}
\hline \multirow[t]{2}{*}{ Exposure } & \multicolumn{2}{|l|}{ Non-adjusted } & \multicolumn{2}{|l|}{ Adjust I } & \multicolumn{2}{|l|}{ Adjust II } & \multicolumn{2}{|l|}{ Adjust III } \\
\hline & HR (95\% CI) & $p$-value & HR (95\% CI) & $p$-value & HR (95\% Cl) & $p$-value & HR (95\% Cl) & $p$-value \\
\hline ABSI $\left(10^{-2} \mathrm{~m}^{11 / 6} \mathrm{~kg}^{-2 / 3}\right)$ & $3.13(2.44,4.01)$ & $<0.001$ & $2.12(1.61,2.79)$ & $<0.001$ & $1.60(1.20,2.14)$ & 0.0013 & $I .5 I(I .13,2.01)$ & 0.005 \\
\hline \multicolumn{9}{|l|}{ ABSI quartile } \\
\hline ABSI QI & I (reference) & & I (reference) & & I (reference) & & I (reference) & \\
\hline ABSI Q2 & $1.79(1.25,2.55)$ & 0.001 & $1.39(0.97,2.00)$ & 0.074 & $1.20(0.83,1.73)$ & 0.323 & $0.97(0.67, \mathrm{I} .4 \mathrm{I})$ & 0.879 \\
\hline ABSI Q3 & $2.58(1.85,3.60)$ & $<0.001$ & $1.77(1.25,2.50)$ & 0.001 & $1.40(0.99,1.99)$ & 0.057 & I.2। $(0.85,1.72)$ & 0.283 \\
\hline ABSI Q4 & $3.26(2.36,4.50)$ & $<0.001$ & $1.99(1.41,2.80)$ & $<0.001$ & $1.43(1.01,2.02)$ & 0.042 & $1.30(0.92,1.83)$ & 0.139 \\
\hline$P$ for trend & $<0.001$ & & $<0.001$ & & 0.035 & & 0.046 & \\
\hline
\end{tabular}

Notes: Data presented are HR $(95 \% \mathrm{Cl})$; Adjust I model adjust for: age, gender; Adjust II model adjust for: Adjust I + smoking status, alcohol intake, BMI, fatty liver, SBP; Adjust III model adjust for: Adjust II + FPG, HbAIc, HDL-cholesterol, triglycerides.

Abbreviation: $A B S I$, a body shape index.

different subgroups. Besides, we found that the HR for incident type 2 diabetes was higher in the participants who are female, which was consistent with the results of Qatar Biobank study and ARIC study. ${ }^{22,24}$ In this study, the proportion of central obesity (WC $>90 \mathrm{~cm}$ in men and $>80 \mathrm{~cm}$ in women) in female was higher than in male (14.9\% versus $11.4 \%)$. The proportions of central obesity in ABSI quartile1 to 4 (female versus male) were $3.7 \%$ versus $2.6 \%, 8.6 \%$ versus $6.5 \%, 17.7 \%$ versus $11.4 \%, 34.9 \%$ versus $22.5 \%$, respectively. As is known, central obesity is an independent risk factors of type 2 diabetes. $^{25,26}$ Maybe it could explain the reason why higher HR were found in female. ABSI appeared to be more strongly related to the risk of T2DM among younger adults than among older adults, which was consistent with Qatar Biobank study. ${ }^{24}$ The similar trends were observed in the association between obesity and the risk of death. ${ }^{27}$ Perhaps it is related to the fact

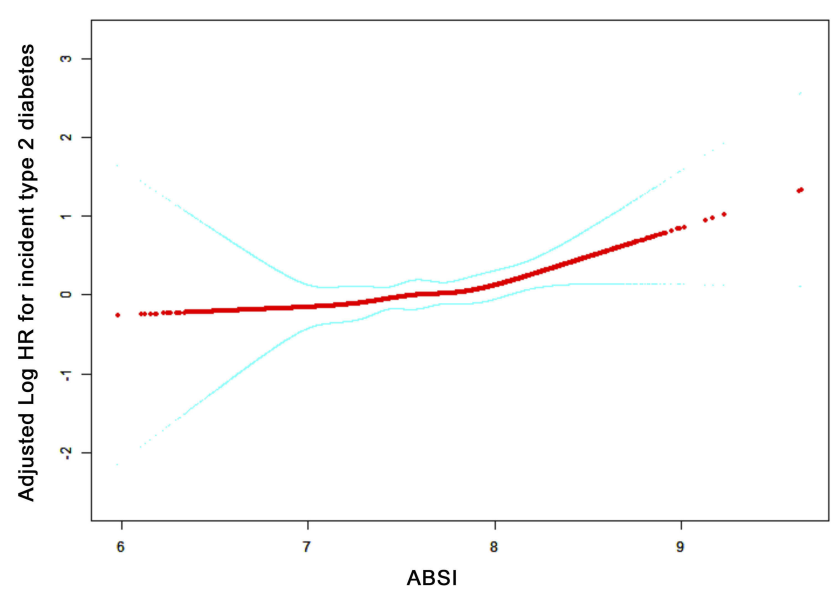

Figure I Dose-response relationship between ABSI and incident type 2 diabetes in the NAGALA study, 2004-2015. In the figure, the red line indicates the estimated risk of incident type 2 diabetes, and the blue lines represent $95 \%$ confidence interval adjusted for age, gender, smoking status, alcohol intake, BMI, fatty liver, SBP, FPG, HbAlc, HDL-cholesterol, and triglycerides.

Abbreviation: $A B S I$, a body shape index. that the old persons paid more attention to their health, and had lower BMI and waist circumference than those of the young (data not shown). We also found participants with overweight or obesity (BMI $\geq 23 \mathrm{~kg} / \mathrm{m}^{2}$ ) had a higher risk of type 2 diabetes compared with normal weight subjects $\left(\mathrm{BMI}<23 \mathrm{~kg} / \mathrm{m}^{2}\right)$. In the Qatar Biobank study, the OR and 95\% CI for diabetes mellitus associated with ABSI were 1.24 (0.66, 2.33), 1.79 (1.30, 2.47), and 2.20 (1.71, 2.83) among normal, overweight, and obese participants. ${ }^{24}$ Besides, the association between ABSI and type 2 diabetes was stronger in participants with fatty liver. Consistently, subgroups with fatty liver or higher BMI had more persons with central obesity. Although our study also showed that ABSI was more strongly related to the risk of T2DM among participants who had never smoked than current smokers, the absolute risk of developing T2DM was higher among current smokers (data not shown). The similar effect of smoking on BMI and risk of death was reported before. ${ }^{27}$

Our study has a number of strengths. First, until now, most of researches about relationship between ABSI and type 2 diabetes are cross-sectional study. This study is a large population-based cohort study, which can reveal the causal relationship. Second, we used strict statistical adjustment to reduce confounding factors. We entered all covariates into a Cox regression model in the basic model or eliminated those covariates in the complete model one by one, and compared the regression coefficients. Those covariates altering initial regression coefficients by more than $10 \%$ were selected. In this study, we adjusted the influence of fatty liver, which presents ectopic fat obesity and is a strong risk factor for incident type 2 diabetes. ${ }^{18}$ Third, to our knowledge, it was the first time to elaborate the linear relationship between ABSI and risk of developing type 


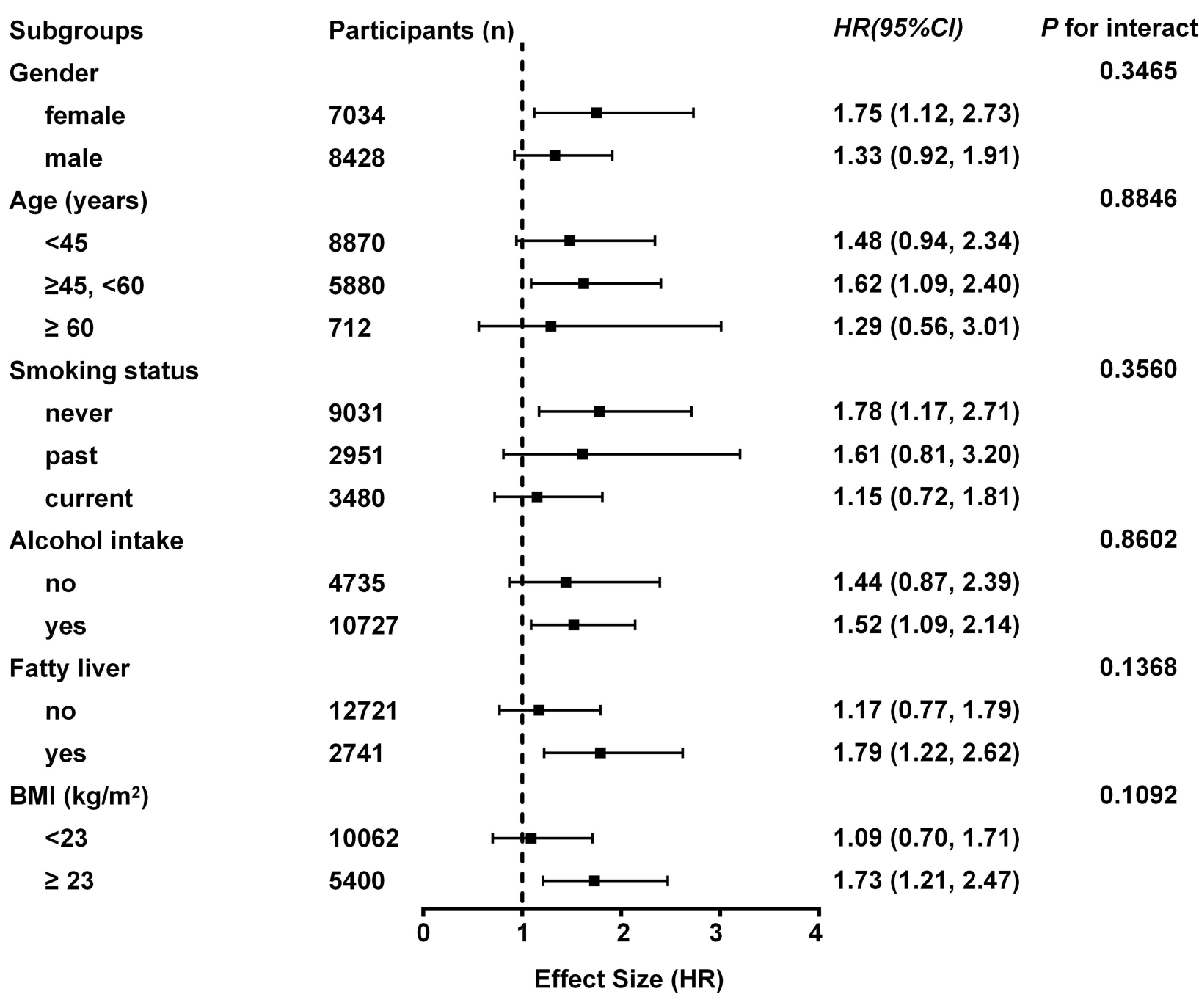

Figure 2 Subgroup analyses of the association between ABSI and incident type 2 diabetes in the NAGALA study, 2004-20I5.

Notes: Adjusted for age, gender, smoking status, alcohol intake, BMI, fatty liver, SBP, FPG, HbAIc, HDL-cholesterol, and triglycerides except the subgroup variable. Abbreviation: BMl, body mass index.

2 diabetes. Fourth, we performed a sensitivity analysis, which made a better use of these data. It showed that the association stably existed in different subgroups.

There are also some limitations in our study. First, due to raw data limitations, we lacked the data on insulin resistance. However, a cross-sectional study showed that the discriminatory power of ABSI for insulin resistance is poor in the general Chinese adults and elderly without diabetes. ${ }^{28}$ We thus thought that lack of insulin resistance would not change hazard rations. Second, diabetes mellitus was not separated into type 1 or type 2 diabetes in this study. Therefore, there was a possibility that the HRs of incident type 2 diabetes were overestimated although the incidence of type 1 diabetes is exceedingly low ( $\sim 2$ cases/year/100,000 individuals) in Japan. ${ }^{29}$ Third, the incidence of type 2 diabetes might have been underestimated because an oral glucose tolerance test was not performed.
Fourth, in this study, we did not exclude participants with pre-diabetes. As the association between ABSI and incident T2DM in participants with pre-diabetes was stronger (data not shown), the HRs for incident T2DM might be overestimated in normal persons. Lastly, a longitudinal ARIC study showed that the effect of ABSI on incident T2DM varied in different race/ ethnicity. $^{22}$ As the study population was only located in Japan, the results could not be generalized.

\section{Conclusions}

In conclusion, our study indicated that a body shape index was associated with an increased risk of type 2 diabetes in Japanese adults, independent of age, gender, smoking status, alcohol intake, BMI, fatty liver, SBP, FPG, HbAlc, HDL-cholesterol, and triglycerides. A positive linear 
association between ABSI and incident type 2 diabetes was observed across the full range of ABSI.

\section{Acknowledgments}

The authors are very grateful to the data providers of the study. They completed the entire study. They are Takuro Okamura, Yoshitaka Hashimoto, Masahide Hamaguchi, Akihiro Obora, Takao Kojima, Michiaki Fukui (corresponding author) (the rankings of these researchers were ranked according to the "reference [18]"). We are very grateful to Xing-Lin Chen for her statistical help.

\section{Disclosure}

The authors declare no conflicts of interest in this work.

\section{References}

1. International Diabetes Federation, IDF diabetes Atlas, International diabetes federation, Brussels, Belgium, 9th edition, 2019. 2019. Available from: http://www.diabetesatlas.org.

2. Chatterjee S, Khunti K, Davies MJ. Type 2 diabetes. Lancet. 2017;389(10085):2239-2251. doi:10.1016/S0140-6736(17)30058-2

3. Vazquez G, Duval S, Jacobs DR, Silventoinen K. Comparison of body mass index, waist circumference, and waist/hip ratio in predicting incident diabetes: a meta-analysis. Epidemiol Rev. 2007;29:115128. doi:10.1093/epirev/mxm008

4. Nyamdorj R, Qiao Q, Lam TH, et al. BMI compared with central obesity indicators in relation to diabetes and hypertension in Asians. Obesity. 2008;16(7):1622-1635. doi:10.1038/oby.2008.73

5. Twig G, Tirosh A, Leiba A, et al. BMI at age 17 years and diabetes mortality in midlife: a nationwide cohort of 2.3 million adolescents. Diabetes Care. 2016;39(11):1996-2003. doi:10.2337/dc16-1203

6. Thomas DM, Bredlau C, Bosy-Westphal A, et al. Relationships between body roundness with body fat and visceral adipose tissue emerging from a new geometrical model. Obesity. 2013;21(11):2264 2271. doi:10.1002/oby.20408

7. Krakauer NY, Krakauer JC. A new body shape index predicts mortality hazard independently of body mass index. PLoS One. 2012;7 (7):e39504. doi:10.1371/journal.pone.0039504

8. He S, Zheng Y, Wang H, Chen X. Assessing the relationship between a body shape index and mortality in a group of middle-aged men. Clin Nutr. 2017;36(5):1355-1359. doi:10.1016/j.clnu.2016.09.003

9. Krakauer NY, Krakauer JC. Dynamic association of mortality hazard with body shape. PLoS One. 2014;9(2):e88793. doi:10.1371/journal. pone. 0088793

10. Song X, Jousilahti P, Stehouwer CD, et al. Cardiovascular and allcause mortality in relation to various anthropometric measures of obesity in Europeans. Nutr Metab Cardiovasc Dis. 2015;25(3):295304. doi:10.1016/j.numecd.2014.09.004

11. Chang Y, Guo X, Chen Y, et al. A body shape index and body roundness index: two new body indices to identify diabetes mellitus among rural populations in northeast China. BMC Public Health. 2015;15:794. doi:10.1186/s12889-015-2150-2

12. Bouchi R, Asakawa M, Ohara N, et al. Indirect measure of visceral adiposity 'A Body Shape Index' (ABSI) is associated with arterial stiffness in patients with type 2 diabetes. BMJ Open Diabetes Res Care. 2016;4(1):e000188. doi:10.1136/bmjdrc-2015-000188

13. Zhao Q, Zhang K, Li Y, et al. Capacity of a body shape index and body roundness index to identify diabetes mellitus in Han Chinese people in Northeast China: a cross-sectional study. Diabet Med. 2018;35(11):1580-1587. doi:10.1111/dme.13787
14. Nascimento-Souza MA, Lima-Costa MF, Peixoto SV. "A body shape index" and its association with arterial hypertension and diabetes mellitus among Brazilian older adults: national health survey (2013). Cad Saude Publica. 2019;35(8):e00175318. doi:10.1590/ 0102-311x00175318

15. Wei J, Liu X, Xue H, Wang Y, Shi Z. Comparisons of visceral adiposity index, body shape index, body mass index and waist circumference and their associations with diabetes mellitus in adults. Nutrients. 2019;11(7):1580. doi:10.3390/ nu11071580

16. Han C, Liu Y, Sun X, et al. Prediction of a new body shape index and body adiposity estimator for development of type 2 diabetes mellitus: the rural Chinese cohort study. Br J Nutr. 2017;118(10):771-776. doi:10.1017/S0007114517002859

17. Okamura T, Hashimoto Y, Hamaguchi M, Obora A, Kojima T, Fukui M. Data from: ectopic fat obesity presents the greatest risk for incident type 2 diabetes: a population-based longitudinal study, dryad, dataset. 2019. Available from: https://datadryad.org/stash/data set/doi:10.5061/dryad.8q0p192.

18. Okamura T, Hashimoto Y, Hamaguchi M, Obora A, Kojima T, Fukui M. Ectopic fat obesity presents the greatest risk for incident type 2 diabetes: a population-based longitudinal study. Int $J$ Obes. 2019;43(1):139-148. doi:10.1038/s41366-018-00 76-3

19. American Diabetes Association. Diagnosis and classification of diabetes mellitus. Diabetes Care. 2013;36(Suppl 1):S67-74. doi:10.23 37/dc13-S067

20. He S, Chen X. Could the new body shape index predict the new onset of diabetes mellitus in the Chinese population? PLoS One. 2013;8(1): e50573. doi:10.1371/journal.pone.0050573

21. Fujita M, Sato Y, Nagashima K, Takahashi S, Hata A. Predictive power of a body shape index for development of diabetes, hypertension, and dyslipidemia in Japanese adults: a retrospective cohort study. PLoS One. 2015;10(6):e0128972. doi:10.1371/journal.pone. 0128972

22. Hardy DS, Stallings DT, Garvin JT, Xu H, Racette SB. Best anthropometric discriminators of incident type 2 diabetes among white and black adults: a longitudinal ARIC study. PLoS One. 2017;12(1): e0168282. doi:10.1371/journal.pone.0168282

23. Vandenbroucke JP, von Elm E, Altman DG, et al. Strengthening the reporting of observational studies in epidemiology (STROBE): explanation and elaboration. PLoS Med. 2007;4(10):e297. doi:10.1371/ journal.pmed.0040297

24. Bawadi H, Abouwatfa M, Alsaeed S, Kerkadi A, Shi Z. Body shape index is a stronger predictor of diabetes. Nutrients. 2019;11(5):1018. doi: $10.3390 /$ nu11051018

25. Frank LK, Heraclides A, Danquah I, et al. Measures of general and central obesity and risk of type 2 diabetes in a Ghanaian population. Trop Med Int Health. 2013;18(2):141-151. doi:10.11 11/tmi. 12024

26. Krishnan S, Rosenberg L, Djoussé L, et al. Overall and central obesity and risk of type 2 diabetes in U.S. black women. Obesity. 2007;15(7):1860-1866. doi:10.1038/oby.2007.220

27. Pischon T, Boeing H, Hoffmann K, et al. General and abdominal adiposity and risk of death in Europe. $N$ Engl $J$ Med. 2008;359 (20):2105-2120. doi:10.1056/NEJMoa0801891

28. Wu K, He S, Zheng Y, Chen X. ABSI is a poor predictor of insulin resistance in Chinese adults and elderly without diabetes. Arch Endocrinol Metab. 2018;62(5):523-529. doi:10.20945/2359-39970 00000072

29. Neville SE, Boye KS, Montgomery WS, et al. Diabetes in Japan: a review of disease burden and approaches to treatment. Diabetes Metab Res Rev. 2009;25(8):705-716. doi:10.1002/dmrr.1 012 


\section{Publish your work in this journal}

Diabetes, Metabolic Syndrome and Obesity: Targets and Therapy is an international, peer-reviewed open-access journal committed to the rapid publication of the latest laboratory and clinical findings in the fields of diabetes, metabolic syndrome and obesity research. Original research, review, case reports, hypothesis formation, expert opinion and commentaries are all considered for publication. The manuscript management system is completely online and includes a very quick and fair peer-review system, which is all easy to use. Visit $\mathrm{http}: / /$ www.dovepress.com/testimonials.php to read real quotes from published authors.

Submit your manuscript here: https:/www.dovepress.com/diabetes-metabolic-syndrome-and-obesity-targets-and-therapy-journal 